\title{
The discrimination of cannabis seed oils and flours by an array of porphyrnoids based gas sensors
}

\author{
R. Risoluti ${ }^{1}$, S. Materazzi ${ }^{1}$, R. Capuano ${ }^{2}$, L. Lvova ${ }^{3}$, C. Di Natale ${ }^{2}$, R. Paolesse ${ }^{3}$ \\ ${ }^{1}$ Department of Chemistry, Sapienza University of Rome, Rome, Italy, \\ ${ }^{2}$ Department of Electronic Engineering, University "Tor Vergata", Rome, Italy, \\ ${ }^{3}$ Department of Chemical Science and Technologies, University "Tor Vergata", Rome, \\ Corresponding author's e-mail address: larisa.Ivova@uniroma2.it
}

\begin{abstract}
:
In this study, we present an application of an array of porphyrinoids-coated quartz microbalances (QMB) gas sensors aimed at distinguishing among derivates of Cannabis Sativa L. plant. To this end, samples of cannabis oil and flour, both commercially available and obtained from local manufactures were tested. Samples were different in terms of cannabinoids, $\Delta 9$-tetrahydrocannabinol (THC) and cannabidiol (CBD), content. Samples composition was determined with a standard GC-MS method. A Partial Least Squares (PLS) Regression model was calculated to estimate the CBD content in cannabis flour. Results show a prediction of the content of CBD with a correlation coefficient $R^{2}=0.961$ and a Root Mean Squared Error of Calibration (RMSEC) of $0.078 \mathrm{mg} / \mathrm{g}$. The results show the Electronic Nose as a promising tool for rapid and non-invasive screening of Cannabis Sativa $L$. products.
\end{abstract}

Key words: Cannabis oil and flour, cannabinoids estimation, Electronic Nose, porphyrinoids-based QMB sensor array.

\section{Introduction}

According to the European legislation a strict control of cannabinoids, such as $\Delta 9$ tetrahydrocannabinol (THC) and cannabidiol (CBD), content in the cannabis (Cannabis Sativa L.) plant derivates, such as oil, flour and tissues is required [1]. The psychotropic THC has a highest affinity to cannabinoid receptors (CB1, CB2) and a great pharmacological and clinical utility; while non-psychotropic CBD has significant health effects and hence the analysis of these compounds in cannabis derivate products is an important issue.

The standard methods to estimate the content of cannabinoids are GC and HPLC [2]. Some other analytical methods, including NMR spectroscopy have been also reported [3]. However, all these techniques employ expensive equipment and reagents, require skilled personnel, complicated (and often destructive) sample pretreatment and long elaboration times. At the moment, in literature and even less in practice, very few examples of non-destructive techniques implementation, such as analysis with chemical sensors and sensor arrays able to distinguish cannabinoids potentially present cannabis plant derivates are described $[4,5]$.
In the present research we report a sensor array based on porphyrinoids-functionalized QMB sensors, for the discrimination of cannabis oil and flour of different formulation. Moreover, the possibility to use the sensor array for quantitative prediction of the cannabinoids amount in Cannabis Sativa $L$. flour was investigated. A satisfactory correlation between the PLS estimation the CBD amount determined by GCMS was found

\section{Experimental}

The sensor array used in the present work is the last version of a series of instruments designed since 1996 at the University of Rome "Tor Vergata". The array is made of 12 AT-cut quartz crystal microbalances (QMB), with a fundamental frequency of $20 \mathrm{MHz}$, coated by different metalloporphyrins and corrole films, among them: free-base 5,10,15,20-tetrakis-(4butyloxy-phenyl)porphyrin ( $\left.\mathrm{H}_{2} \mathrm{TPP}\right), 5,10,15,20$ tetrakis-(4-butyloxyphenyl)porphyrinates of copper(II) (TBPCu), zinc(II) (TBPZn), cobalt(II) ( TBPCo), magnesium(II) (TBPMg), iron(III) chloride (TBPFeCl), manganese(III) chloride (TBPMnCl) and tin(IV) dicloride ( $\mathrm{TBPSnCl}_{2}$ ); free-base 5,10,15-tritolyl-corrole $\left(\mathrm{H}_{3} \mathrm{TTC}\right)$, 5,10,15-tritolyl-corroles of copper(III) (CUTTC), 
manganese(III) chloride (TCCMnCl) and dihydroxy phosporus (TTCP $\left.(\mathrm{OH})_{2}\right)$. In QMB sensors the adsorption of volatile compounds onto the quartz surface results in a frequency shift $(\Delta f)$ proportional to the mass variation. The baseline of sensor signals was measured using a constant flow of ambient air filtered with a $\mathrm{CaCl}_{2}$ trap. The difference of the sensors signals taken in reference air respect to sample air was used as the sensor response. The headspace of the considered samples was analyzed for 3 minutes at the constant flow of $50 \mathrm{sccm}$. Two cannabis seed oil samples (one commercial sample and one obtained from local obtained manufacture) and three commercially available samples of cannabis flour were tested in tree replicates for each sample. $1 \mathrm{~g}$ of flour sample or $500 \mu \mathrm{l}$ of oil were placed inside sealed vials and the analysis of samples headspace was performed with Electronic Nose at room temperature $\left(20^{\circ} \mathrm{C}\right)$ or after heating at $70^{\circ} \mathrm{C}$ for 5 min. Independently samples were analyzed by CG-MS method for content of THC and CBD. Principal Component Analysis (PCA) and PLS regression were applied to discriminate among the cannabis derivate samples. The PLS model was validated by a leave-one-out crossvalidation. The RMSEC and RMSEV (Root Mean Square Error of Calibration and Validation respectively), and the correlation coefficient of predicted versus measured correlation line, $R^{2}$, were used to evaluate the efficiency of obtained PLS models. All chemometric analysis was performed with Unscrambler software (v. 9.7, 2007, CAMO Software AS, Norway).

\section{Results and Discussion}

All samples contained low amount of psychotropic THC (lower than LOD of CG-MS) on the background of much higher amount of coexisting CBD.

Tab. 1: The results of GC-MS analysis of cannabis derivate products.

\begin{tabular}{|c|c|c|}
\hline sample & CBD, $\%$ wv & St. dev, CBD, \% \\
\hline Oil1 & 0.150 & 0.074 \\
\hline Oil1 & 3.43 & 1.08 \\
\hline Flour 1 & 0.157 & 0.005 \\
\hline Flour 2 & 0.144 & 0.001 \\
\hline Flour 3 & 0.065 & 0.005 \\
\hline
\end{tabular}

The locally produced Oil2 is characterized by higher percentages of cannabinoids, and this difference was well captures by the sensors. Fig. 1. shows the scores plot of the first two principal components. $99 \%$ of the total variance is explained in the plot where a clear separation among samples is observed.

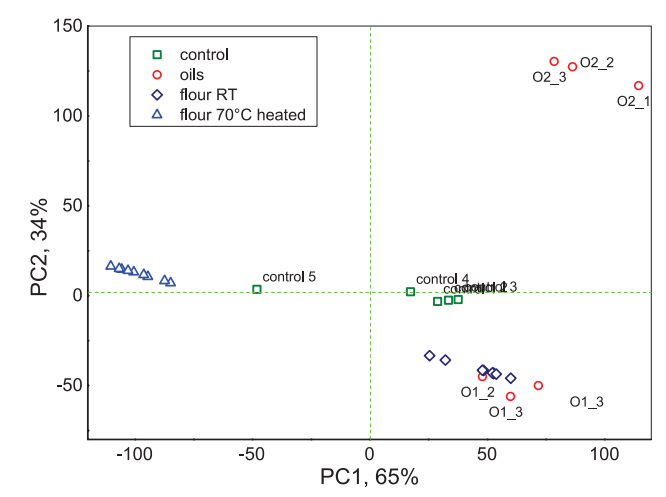

Fig. 1. PCA score plot of Electronic Nose analysis of cannabis oil and flour.

Furthermore, a PLS regression model was calculated in order to estimate the CBD amount in cannabis flour. The content measured with the standard GC-MS was used as a calibration reference. The correlation coefficient between PLS estimate and calibration reference was $\mathrm{R}^{2}=0.961$ with a $\mathrm{RMSEC}$ of $0.078 \mathrm{mg} / \mathrm{g}$.

These results demonstrate the potential utility of the porphyrinoids based sensor array as a rapid non-invasive screening of cannabinoids. Furthermore, the sensors measurement is performed at room temperature and it does not require any sample pretreatment. The further research on discrimination of cannabis derivates products is now in the progress in our laboratories.

\section{References}

[1] Official Journal L $332,28 / 12 / 2000$ P. 0063 0075, Commission Regulation (EC) No 2860/2000 of 27 December 2000, http://data.europa.eu/eli/reg/2000/2860/oj.

[2] W. Peschel, M. Politi, Talanta 140, 150-165 (2015); doi: 10.1016/j.talanta.2015.02.040.

[3] R. Jain, R. Singh, Trends in Analytical Chemistry 80, 156-166 (2016); 10.1016/j.trac.2016.03.012.

[4] Z. Haddi, A. Amari, H. Alami, N. El Bari, E. Llobet, B. Bouchikhi, Sens. Actuators B 155, 456-463 (2011); doi: 10.1016/j.snb.2010.12.047.

[5] A. Voss, K. Witt, T. Kaschowitz, W. Poitz, A. Ebert, P. Roser, K.-J. Bär, Sensors 14, 1325613272 (2014); doi:10.3390/s140713256. 